\title{
Editorial
}

Schmerz 2015 $\cdot 29: 465$

DOI 10.1007/s00482-015-0066-5

Online publiziert: 21. September 2015

(c) Deutsche Schmerzgesellschaft e.V. Published by Springer-Verlag Berlin Heidelberg - all rights reserved 2015

CrossMark

\section{R.-D. Treede}

Zentrum für Biomedizin und Medizintechnik Mannheim, Medizinische Fakultät

Mannheim der Universität Heidelberg, Mannheim, Deutschland

\section{Deutsche Schmerzgesellschaft - erfolgreich in Deutschland und der Welt}

Die Deutsche Schmerzgesellschaft ist eine der ältesten nationalen Schmerzgesellschaften in der „Familie“ der International Association for the Study of Pain (IASP). Sie hat im Verlauf von vier Jahrzehnten eine große Zahl von Mitgliedern aus Anästhesiologie, Psychologie, Neurologie, Orthopädie, Grundlagenforschung, Pflege, Physiotherapie und anderen Fächern und Berufsgruppen gewinnen können und ist damit nach der Chinesischen Schmerzgesellschaft die zweitgrößte Schmerzgesellschaft weltweit. Grundlagenforscher und klinische Forscher aus Deutschland haben viele wichtige Beiträge zur Literatur über Mechanismen und Behandlung akuter und chronischer Schmerzen publiziert, zunächst vor allem zur Neurophysiologie peripherer Nozizeptoren, später dann auch zu allen anderen Aspekten. In der IASP-Zeitschrift PAIN ${ }^{\circledR}$ kommen seit vielen Jahren nach den USA die meisten Beiträge aus Deutschland.

Mit der Einführung der Weiterbildung „Spezielle Schmerztherapie“ und später den Querschnittsbereichen 13 „Palliativmedizin“ und 14 „Schmerzmedizin“ im Medizinstudium hat sich die Deutsche Schmerzgesellschaft in Aus- und Weiterbildungsfragen der Medizin weltweit an die Spitze gesetzt. In der praktischen Ausübung der Schmerzmedizin und der flächendeckenden Versorgung der Bevölkerung sind sicher noch etliche Fragen offen und noch nicht alle Konzepte umgesetzt. Trotzdem kann man festhalten, dass es die Deutsche Schmerzgesellschaft in ihrer Geschichte weit gebracht hat und dass aller Anlass besteht anzunehmen, dass in Deutschland nicht nur die Vision der IASP (www.iasp-pain.org) umgesetzt wird

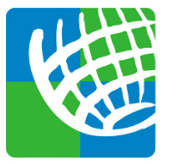

International Association for the Study of Pain

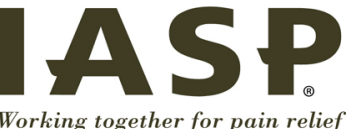

Working together for pain relief

》) "Working together for pain relief throughout the world"

sondern dass die Errungenschaften der Schmerzmedizin mehr und mehr auch bei den Patienten ankommen werden.

Herzlichen Glückwunsch zum Geburtstag!

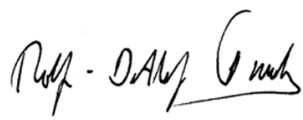

R.-D. Treede

Präsident der IASP (2014-2016)

\section{Korrespondenzadresse}

Prof. Dr. R.-D. Treede

Zentrum für Biomedizin und Medizintechnik Mannheim

Medizinische Fakultät Mannheim der Universität Heidelberg

Ludolf-Krehl-Str. 13-17, 68167 Mannheim rolf-detlef.treede@medma.uni-heidelberg.de 